\title{
Attractiveness and distinctiveness in voices and faces of young adults
}

Authors: Romi Zäske (corresponding author)

Department for General Psychology and Cognitive Neuroscience \& DFG Research Unit Person Perception, Institute of Psychology, Friedrich Schiller University of Jena, Am Steiger 3/1, 07743 Jena, Germany

Department of Otorhinolaryngology, Jena University Hospital, Am Klinikum 1, 07747, Jena, Germany

Tel.: +49-3641-945 935, Email: romi.zaeske@uni-jena.de

\section{Verena Gabriele Skuk}

Department of Otorhinolaryngology, Jena University Hospital, Am Klinikum 1, 07747, Jena, Germany, Tel.: +49-3641-945 939, Email:

verena.g.skuk@gmail.com

\section{Stefan R. Schweinberger}

Department for General Psychology and Cognitive Neuroscience \& DFG Research Unit Person Perception, Institute of Psychology, Friedrich Schiller University of Jena, Am Steiger 3/1, 07743 Jena, Germany,

Tel.: +49-3641-945 182, Email: stefan.schweinberger@uni-jena.de 
Abstract: Facial attractiveness has been linked to the averageness (or typicality) of a face. More tentatively, it has also been linked to a speaker's vocal attractiveness, via the "honest signal" hypothesis, holding that attractiveness signals good genes. In four experiments, we assessed ratings for attractiveness and two common measures of distinctiveness ("distinctiveness-in-thecrowd"-DITC and "deviation-based distinctiveness"-DEV) for faces and voices (vowels or sentences) from 64 young adult speakers (32 female). Consistent and strong negative correlations between attractiveness and DEV generally supported the averageness account of attractiveness for both voices and faces. By contrast, indicating that both measures of distinctiveness reflect different constructs, correlations between attractiveness and DITC were numerically positive for faces (though small and non-significant), and significant for voices in sentence stimuli. As the only exception, voice ratings based on vowels exhibited a moderate but significant negative correlation between attractiveness and DITC. Between faces and voices, distinctiveness ratings were uncorrelated. Remarkably, and at variance with the honest signal hypothesis, vocal and facial attractiveness were uncorrelated, with the exception of a moderate positive correlation for vowels. Overall, while our findings strongly support an averageness account of attractiveness for both domains, they provide little evidence for an honest signal account of facial and vocal attractiveness in complex naturalistic speech. Although our findings for vowels do not rule out the tentative notion that more primitive vocalizations can provide relevant clues to genetic fitness, researchers should carefully consider the nature of voice samples, and the degree to which these are representative of human vocal communication.

Key words: voice; face; attractiveness; distinctiveness; averageness; honest signal hypothesis 


\section{Introduction}

The question of what constitutes attractiveness in a human face or a voice has concerned scientists for a long time (e.g., Cunningham, 1986; Zuckerman \& Miyake, 1993). Contemporary research suggested a substantial role of averageness for perceptions of attractiveness. Specifically, digitally averaged faces are perceived as more attractive the more single faces are entered into an average (Langlois \& Roggman, 1990; but see also Perrett et al., 1998, on the role of sexual dimorphism). At the same time averaging decreases distinctiveness by normalizing features that distinguish individual faces from one another. Valentine's influential face space framework (Valentine, 1991) holds that all faces we encounter are stored within a multidimensional space relative to an average prototypical face. Accordingly, a given face is more distinctive the more it deviates from the average in terms of its feature dimensions. Similarly, averaging voices across increasing numbers of speakers was more recently reported to enhance perceived vocal attractiveness, at least for simple vocalizations such as vowels or syllables (Andraszewicz, Yamagishi, King, \& Ieee, 2011; Bruckert et al., 2010) and to decrease perceived vocal distinctiveness (Latinus, McAleer, Bestelmeyer, \& Belin, 2013). In these studies, vocal attractiveness and distinctiveness ratings correlated with the location of voices in a putative multidimensional "voice-space" in which dimensions reflected acoustic properties such as fundamental frequency (f0; Andraszewicz et al., 2011; Bruckert et al., 2010; Latinus et al., 2013), the first formant (F1; Andraszewicz et al., 2011; Bruckert et al., 2010), formant dispersion (Latinus et al., 2013), or harmonics-to noise ratio (Andraszewicz et al., 2011; Bruckert et al., 2010; Latinus et al., 2013). Although these effects could in part be due to a preference for "typical" stimuli (Babel \& McGuire, 2015; Babel, McGuire, \& King, 2014; but see Collins, 2000; Feinberg, DeBruine, Jones, \& Perrett, 2008; Jones, Feinberg, DeBruine, Little, \& Vukovic, 2010 on the role of sexual dimorphism for vocal attractiveness) and a reduction of distinctiveness by averaging, the relationship between attractiveness and distinctiveness has been rarely studied directly, particularly in the case of voice perception. 
Of relevance, the relationship of vocal attractiveness and distinctiveness appears to be affected by the speech material used. Specifically, while for simple vowel stimuli perceived attractiveness was reported to correlate negatively with the deviation of acoustic measures from the average (Andraszewicz et al., 2011; Bruckert et al., 2010) no correlation was found between these measures and attractiveness ratings for more complex word or sentence stimuli (Andraszewicz et al., 2011). On the one hand, this may be surprising considering that other speaker attributes can usually be discerned across various utterances of a given speaker such as speaker identity (Skuk \& Schweinberger, 2013), sex (Fellowes, Remez, \& Rubin, 1997; Lass, Hughes, Bowyer, Waters, \& Bourne, 1976), or age (Linville, 1996). On the other hand, there is accumulating evidence that the processing of linguistic and non-linguistic properties of voices, i.e. speech content and speaker attributes, are not entirely independent (for recent reviews see Kreiman \& van Lancker Sidtis, 2011; Schweinberger, Kawahara, Simpson, Skuk, \& Zäske, 2014). It is therefore of further interest in the present study how speech materials (in the present case, simple vowels vs. more complex naturalistic sentences) may affect the relationship of perceived attractiveness and distinctiveness in voices and faces.

In the case of faces, distinctiveness has been assessed by two measures: One measure of facial distinctiveness (face-in-the-crowd, or FITC distinctiveness) involves asking raters how easily they would spot a face in a group of people. A second measure involves asking raters to judge how much a face deviates from other known faces (deviation-based, or DEV distinctiveness). Although these measures are often taken to reflect the same construct, a recent study on a large set of 560 faces suggested that both measures only correlate to a very moderate extent (Wiese, Altmann, \& Schweinberger, 2014). Strikingly, while there was a negative correlation between DEV distinctiveness and attractiveness, there was a positive correlation between FITC distinctiveness and attractiveness. A similarly detailed study on the relationship between distinctiveness and attractiveness of voices is unavailable as of yet. One interpretation of the above findings suggests that the deviation-based measure of distinctiveness is the more 
valid measure in terms of Valentine's model (Valentine, 1991), and that the FITC rating might be distorted by cognitive heuristics such as "Surely I would spot such an attractive face", thus biasing FITC ratings for extremely attractive faces towards higher FITC distinctiveness (Wiese et al., 2014). If so, we would expect analogous patterns of relationships between measures of vocal distinctiveness and attractiveness.

Perceived attractiveness is often taken as a signal for genetic fitness or health, (e.g. Feinberg, 2008; Rhodes, 2006). For instance, physical features of both faces and voices could be influenced by common (e.g. hormonal) factors, and accordingly might provide "honest signals" to mate value (Feinberg, 2008; Little, Connely, Feinberg, Jones, \& Roberts, 2011). If so, such common factors could provide a basis for positive correlations between facial and vocal attractiveness in the same individual. Indeed, a few studies found vocal and facial attractiveness of women to be correlated (Abend, Pfluger, Koppensteiner, Coquerelle, \& Grammer, 2015; Collins \& Missing, 2003; Valentova, Varella, Havlicek, \& Kleisner, 2017). Moreover, vocal and visual attractiveness of twelve male speakers were also found to be correlated when rated by either adolescents or adults (but not when rated by children: Saxton, Caryl, \& Roberts, 2006). Others found a correlation only after aggregating data across female and male speakers (Rezlescu et al., 2015). However, voice ratings were typically based on vowels or single words (Collins \& Missing, 2003; Rezlescu et al., 2015; Saxton et al., 2006), such that the relationship between vocal and facial attractiveness has never been compared for different types of utterances. Note that two studies suggest that positive correlations between ratings for faces and voices of women (Feinberg et al., 2005) and men (Feinberg, DeBruine, Jones, \& Little, 2008) could be based on sexual dimorphism, rather than prototypicality or averageness per se, as an aspect of attractiveness (c.f. Rhodes, 2006). The consideration of utterance type in voices may be important since isolated vowels and sentences differ substantially in the amount and type of phonetic information they convey. Sentences in particular convey information not only about biophysical inevitabilities of an individual speaker, but also about socially acquired voice 
characteristics which determine, for instance, phonetic or prosodic differences between male and female voices (Simpson, 2009). Therefore, it is reasonable that the type of utterance also affects personal impressions of attractiveness and distinctiveness (for a similar argument see Babel \& McGuire, 2015).

In the present study, we obtained photographs and voices from a set of young adult speakers, and obtained ratings of attractiveness and two measures of distinctiveness both for vowel stimuli (Exp. 1 and 2) and sentence stimuli (Exp. 3 and 4). Our first aim was to determine whether a pattern of relationships between measures of distinctiveness and attractiveness that is similar to what has been reported recently for faces (Wiese et al., 2014) would also emerge for voices. Our second aim was to investigate the degree of cross-domain relationships between measures of attractiveness and distinctiveness for the voices and faces of the same speakers, while considering potential effects for different types of utterances.

\section{Methods}

\subsection{Recording}

\subsubsection{Speakers}

We recruited 64 young speakers (32 female, $M=21.9$ yrs, aged 18-25 yrs) among students of the University of (location of university will be provided after anonymous review). Age of female and male speakers did not differ significantly $(t[62]=-0.392, p=.696)$. All speakers received $€ 10$ for participation and gave their written informed consent to the use of their faces and voices as stimuli in the current and future experiments..

\subsubsection{Stimuli}

From each speaker we took photographs and recorded sustained vowels ([a:],[e:],[i:],[o:],[u:]) and German sentences (sentence \#1: "Keine Antwort ist auch eine Antwort" [No answer is an answer too.]; sentence \#2: "Magdala liegt bei Apolda" [Magdala is 
near Apolda.]; sentence \#3: "Die Oma mag Urlaub am Meer." [Grandma likes to holiday by the sea.]) in a quiet and semi-anechoic room, as part of a more extensive protocol. The recording room was situated in a quiet tract of the building and did not have any windows. Walls, ceiling and floor of this room were fitted with molton, to eliminate reverberation. To standardize photographs, speakers sat on a chair in front of a green background and were illuminated by a three-point lighting system. All wore a black cape around their shoulders, took off glasses, jewelry and make-up and men shaved before the session. Portraits were captured with a Sony DCR-DVD403E camcorder. Using Adobe Photoshop CS5TM, faces were cropped, standardized in size and vertical eye position, and pasted on a grey background.

To avoid gross differences in articulation style for voice recordings that might potentially affect ratings irrespective of voice quality, speakers were asked to produce vowels and sentences in similar style, intensity and timing, and in emotionally neutral intonation, as exemplified in a pre-recorded model speaker (first author, presented via loudspeakers). Voices were recorded with a Sennheiser MD 421-II microphone with a pop protection and a Zoom H4n audio interface (16-bit resolution, $48 \mathrm{kHz}$ ). The interface was connected to a computer in the neighboring room at which the audio manager monitored recordings via Adobe Audition 3.0. Each utterance was recorded four to five times to choose the best audio recordings (no artifacts and clear articulation) as stimuli. Using PRAAT (Boersma \& Weenink, 2001), vowels were cut to contain the stable phase (duration $1500 \mathrm{~ms}$ ) and sentences were cut at first word onset and last word offset (sentence durations: \#1: $M=2358 \mathrm{~ms}, S D=245$, range 1833 - $3081 \mathrm{~ms}$; \#2: $M$ $=1979 \mathrm{~ms}, S D=180$, range $1583-2671 \mathrm{~ms} ; \# 3: M=1883 \mathrm{~ms}, S D=133$, range $1620-2207$ ms). All stimuli were converted to mono, and were RMS normalized to 70dB. In order to fade vowel stimuli in and out the first and last $180 \mathrm{~ms}$ were multiplied with a cosine window function. 


\subsection{Rating}

\subsubsection{Raters}

Four groups of twenty (ten female) student participants each contributed data to Experiments 1 to 4 respectively (Exp. 1: 4 left-handed, $M=21.7$ yrs, range 19-28; Exp. 2: all right-handed, $M=23.5$ yrs, range 18-30; Exp. 3: 6 left-handed, $M=22.4$ yrs, range 18-30; Exp. 4: 2 left-handed, $M=22.3 \mathrm{yrs}$, range 19-27). Data from five additional participants were excluded from the analyses (Exp. 1: $N=3$; Exp. 3: $N=1$; Exp. 4: $N=1$ ) due to technical problems or task incompliance $(N=3)$, or extremely low variance of responses $(S D<.60 ; N=$ 2). All participants were native speakers of German and none reported hearing difficulties. Participants received a payment of $€ 5$ or course credit. All gave written informed consent. The study was conducted in accordance with the Declaration of Helsinki, and was approved by the Faculty Ethics Committee of the University of (location of University will be provided after anonymous review).

\subsubsection{Procedure}

Raters were tested individually in a quiet sound-attenuated chamber, situated within the experimenter's room. Instructions were presented in writing on a computer screen. Voice stimuli were presented diotically via Sennheiser HD 212Pro over-ear headphones at a constant and comfortable intensity, with an approximate peak intensity of $70 \mathrm{~dB}(\mathrm{~A})$ as determined with a Brüel \& Kjær Precision Sound Level Meter Type 2206. Face stimuli subtended a visual angle of $\sim 10.9^{\circ}$ vertical $\times 7.9^{\circ}$ horizontal, at a viewing distance of $65 \mathrm{~cm}$. Voices and faces were rated blockwise on 6-point rating scales, with sub-blocks for attractiveness ratings and distinctiveness ratings. Depending on experiment, distinctiveness was measured as either "distinctiveness-inthe-crowd" (DITC) for voices/faces (VITC/FITC, Exp. 1 and 3), or as deviation (DEV) from the mean (Exp. 2 and 4). Individual trials used single presentations either of one of five vowels (Exp. 1 and 2), or of one of three sentences (Exp. 3 and 4) per speaker. Sub-blocks for attractiveness and distinctiveness ratings were further subdivided into male and female stimulus 
blocks, each of which contained either 32 face trials (i.e. one trial per speaker) or a number of voice trials depending on the experiment: while there were 160 voice trials (32 speakers $\mathrm{x} 5$ vowels) per male and female rating block in Exp. 1 and 2, 96 trials were presented in Exp. 3 and 4 (32 speakers x 3 sentences). Within male and female blocks, trials were always presented in randomized order. Across participants block order was balanced for modality (face or voice), rating task (attractiveness or distinctiveness) and stimulus sex (male or female). Specifically, one group of participants first rated faces for attractiveness starting with male faces, followed by female faces, then rated distinctiveness of male and female faces, and finally performed voice ratings in the analogous block order as for faces. In another group the block order was swapped, such that participants first rated voices for distinctiveness starting with female voices, followed by male voices, then rated attractiveness of female and male voices, and finally rated faces according to the same block order. Rating instructions for the various tasks were as follows (with German translations in parentheses): Attractiveness: "Please assess how attractive/unattractive the voices are, in the sense of sounding pleasant" (Beurteilen Sie bitte nun, wie attraktiv/unattraktiv die Stimmen sind. Attraktivität ist hier gemeint im Sinne von "wohlklingend".) or "Please assess how attractive/unattractive the faces are, in the sense of good-looking" (Beurteilen Sie bitte nun, wie attraktiv/unattraktiv die Gesichter sind. Attraktivität ist hier gemeint im Sinne von "gutaussehend".); VITC: "Please assess how distinctive the voices are. Imagine yourself on a busy square. You are surrounded by many people who are talking simultaneously. A voice is distinctive if it stands out of the crowd" (Beurteilen Sie bitte die Stimmen danach, wie distinkt sie klingen. Stellen Sie sich dazu vor, dass Sie auf einem belebten Platz stehen. Sie sind umgeben von vielen Personen, die durcheinander reden. Eine Stimme ist distinkt, wenn sie aus der Menge heraussticht.) or FITC: "Please assess how distinctive the faces are. Imagine yourself on a busy square. You are surrounded by many people. A face is distinctive if it stands out of the crowd" (Beurteilen Sie bitte die Gesichter danach, wie distinkt sie aussehen. Stellen Sie sich dazu vor, dass sie auf einem belebten Platz stehen. Sie sind umgeben von vielen Gesichtern. Ein Gesicht ist distinkt, 
wenn es aus der Menge heraussticht.). DEV: "Please assess how typical/untypical the voices/faces are. Ask yourself how much the respective voice/face differs from other voices/faces you know." (Beurteilen Sie bitte die Stimmen/Gesichter danach, wie typisch/untypisch sie sind. Fragen Sie sich dazu, wie stark sich die jeweilige Stimme/das jeweilige Gesicht von anderen Ihnen bekannten Stimmen/Gesichtern unterscheidet.)

Finally, we recorded familiarity judgments for faces, to rule out prior familiarity with the speakers. The experiment lasted 60 min. Trials started with a black central fixation cross on a grey background with a reminder of the task and response alternatives below. After $1000 \mathrm{~ms}$ the cross was either joined by a voice with a constant duration for vowels in Exp. 1 and 2 (1500 ms) and variable duration for sentences in Exp. 3 and 4 (cf. 2.1.2 Stimuli), or replaced by a face which remained on screen until a response was entered. This means that whereas voice stimuli were presented for the duration of the utterance (between about 1.5 and $3.1 \mathrm{~s}$, depending on utterance), face stimuli remained on screen until a response was given. Note that after about 100 ms of exposure duration, evaluations of faces appear to remain remarkably stable even for much longer exposure durations (Willis \& Todorov, 2006). Accordingly, while inherent differences in timing at which social information unfolds in static faces as compared to voices preclude a fair comparison, such procedural differences are unlikely to have affected the present findings. There was no time limit for the responses, but participants were encouraged to respond spontaneously and as accurately as possible. Ratings (six-point scales, with $1=$ very unattractive/undistinctive/typical and $6=$ very attractive/distinctive/untypical) and 2-AFC familiarity judgments ("d" or "l" for familiar vs. unfamiliar) were entered via a computer keyboard.

\section{Results}

For each experiment, we computed Spearman rank correlations between mean attractiveness and distinctiveness ratings (per speaker, averaged across raters) within voice and face domains (cf. Fig 1), as well as between domains (cf. Fig 2). Based on face familiarity 
judgments, we excluded a small percentage of ratings for familiar speakers from these analyses (3.4\%, $2.0 \%, 1.5 \%$, and $1.1 \%$ of trials for Exp. 1 - 4 respectively). On average, each stimulus thereby obtained $19.3 / 20(S D=0.9$, range $15-20), 19.6 / 20(S D=0.6$, range $18-20), 19.7 / 20(S D$ $=0.5$, range 18-20), and 19.8/20 $(S D=0.5$, range 18-20) of valid ratings in Exp. $1-4$ respectively. For the calculation of interrater-reliability, i.e. the agreement between raters on attractiveness and distinctiveness of faces and voices, Cronbach's $\alpha$ was computed (with ratings for each speaker collapsed across individual vowels or sentences). To this end, ratings for speakers with whom raters had indicated familiarity $(3.4 \%, 2.0 \%, 1.5 \%$, and $1.1 \%$ of trials for Exp. 1 - 4 respectively) were replaced by mean ratings per participant across all speakers (thus, leading to more conservative, i.e. lower reliability estimates). Interrater-reliability was generally high $(.763 \leq \alpha<.923)$ except for DITC ratings for voices based on vowel utterances $(.640 \leq \alpha<$ .647, cf. supplemental Table S1).

For correlations within and between voice and face domains, voice ratings were averaged across the five vowels (Exp. 1 and 2) and across the three sentences (Exp. 3 and 4). Due to technical issues one sound sample (sentence \#1) of a female speaker (fKL23) in Exp. 3 and 4 failed to be presented, such that mean ratings of that speaker's voice were based on two, instead of three, sentences. This was done to aggregate across more trials in order to obtain more stable ratings per speaker, and because initial analyses had suggested consistently positive correlations between both individual vowels, and between individual sentences.

Within domains (Table 1, Fig. 1), we obtained a significant negative correlation between DITC and attractiveness ratings in Exp. 1 when ratings for voices were based on vowels $(\rho[62]=-.45, p<.001)$. Accordingly, voices rated as more distinctive in VITC ratings were rated as less attractive. For faces, no negative correlation between FITC and attractiveness was found; to the contrary, we observed a marginal positive correlation $(\rho[62]=.21, p=.101)$. In Exp. 2, strong negative correlations between DEV and attractiveness were seen both for voices based on vowels $(\rho[62]=-.85, p<.001)$ and for faces $(\rho[62]=-.64, p<.001)$. In Exp. 3, a small positive correlation between DITC and attractiveness was seen for voices based on 
sentences $(\rho[62]=.28, p=.028)$, but again no significant correlation emerged for faces $(\rho[62]=$ $-.06, p=.660)$. In Exp. 4, strong negative correlations between DEV and attractiveness were seen both for voices based on sentences $(\rho[62]=-.87, p<.001)$ and, as in Exp. 2, for faces $(\rho[62]=-.74, p<.001)$.

To explore possible effects of the type of utterance on the above within-domain correlations, we also computed correlations for voices separately for each vowel (Exp. 1 and 2) and sentence (Exp. 3 and 4). Details can be found in supplemental Tables S2 - S5. In short, these analyses indicate that the negative correlations between voice attractiveness and DEV were consistently present across vowels and sentences throughout (supplemental Tables S3 and S5, Exp. 2 and 4). In contrast, both the negative correlations between voice attractiveness and DITC for vowels (supplemental Table S2, Exp. 1) and the positive correlations between voice attractiveness and DITC for sentences (supplemental Table S4, Exp. 3) were less consistently seen across voice samples, suggesting a role of individual utterances in addition to an effect of speaker per se. For the interested reader we report within-domain correlations also separately for male and female raters (supplemental Tables S10 and S11). In short, these analyses suggest similar results for both male and female raters. We also report statistical comparisons of the magnitudes of correlations for female vs. male speakers (supplemental Table S14), which generally show similar correlations as well. The only exception were significantly larger negative correlations between face attractiveness and DEV for female compared to male faces, in both Exp. 2 and 4. Of note, negative correlations remained significant and highly consistent for male stimuli when tested separately, indicating a qualitatively similar, albeit relatively weaker, relationship. 
Table 1. Spearman $\rho$ correlation coefficients (and p-values) for within-domain (voice and face) correlations between attractiveness and distinctiveness ratings (DITC in Exp.1 and 3; DEV in Exp. 2 and 4) depicted separately for female, male and all voices and faces in Exp. 1 - 4.

Vowels

Sentences

\begin{tabular}{|c|c|c|c|c|c|c|c|c|}
\hline \multirow[t]{3}{*}{ Speaker Sex } & \multicolumn{2}{|c|}{ Voices } & \multicolumn{2}{|c|}{ Faces } & \multicolumn{2}{|c|}{ Voices } & \multicolumn{2}{|c|}{ Faces } \\
\hline & \multicolumn{4}{|c|}{ Experiment 1 (DITC) } & \multicolumn{4}{|c|}{ Experiment 3 (DITC) } \\
\hline & -.412 & $(.019)$ & .229 & $(.207)$ & .432 & $(.013)$ & -.071 & (.698) \\
\hline Male & -.324 & $(.070)$ & .128 & $(.484)$ & .119 & $(.515)$ & -.089 & (.628) \\
\hline \multirow[t]{2}{*}{ All } & -.450 & $(<.001)$ & .207 & (.101) & .275 & (.028) & -.056 & (.660) \\
\hline & \multicolumn{4}{|c|}{ Experiment 2 (DEV) } & \multicolumn{4}{|c|}{ Experiment 4 (DEV) } \\
\hline Female & -.842 & $(<.001)$ & -.804 & $(<.001)$ & -.906 & $(<.001)$ & -.939 & $(<.001)$ \\
\hline Male & -.860 & $(<.001)$ & -.496 & $(.004)$ & -.804 & $(<.001)$ & -.580 & $(.001)$ \\
\hline All & -.853 & $(<.001)$ & -.639 & $(<.001)$ & -.866 & $(<.001)$ & -.744 & $(<.001)$ \\
\hline
\end{tabular}
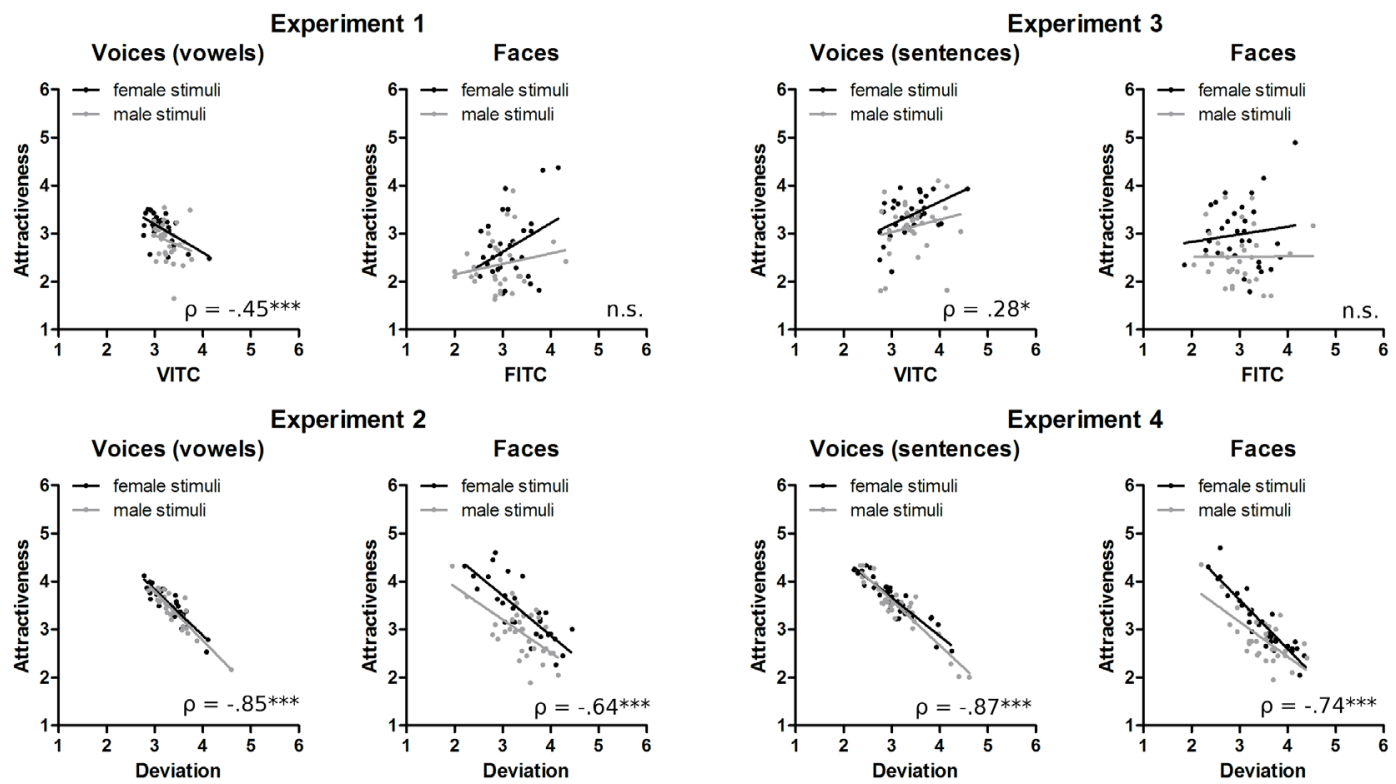

Fig 1. Within-domain correlations between mean ratings of attractiveness and distinctiveness (top: DITCbased distinctiveness; bottom: deviation-based distinctiveness) for faces and voices based on vowels (left: Exp. 1 and 2) and based on sentences (right: Exp. 3 and 4). Data points represent individual speakers, and are depicted separately for female (black) and male speakers (grey). Correlation coefficients $(\rho)$ are depicted overall, with asterisks indicating significance levels $\left({ }^{*} p<.05 ; * * p .01 ; * * *<.001\right)$. 
Between domains (Table 2, Fig. 2), we generally observed no significant correlations between vocal and facial attractiveness for Exp. $2-4(.09<\rho[62]<.24 ; .059<p<.476)$. The only exception was a small but significant positive correlation between vocal and facial attractiveness ratings in Exp. 1, when voice ratings were based on vowel stimuli $(\rho[62]=.30, p$ $=.014)$. Moreover, no significant between-domain correlations were found for distinctiveness ratings in any of the experiments $(-.21<\rho[62]<.18 ; .095<p<.476)$. Between-domain correlations were also computed separately for vowels and sentences in the respective experiments (see supplemental Tables S6 - S9).

Table 2. Spearman $\rho$ correlation coefficients (and p-values) for between-domain (voice-face) correlations depicted separately for attractiveness and distinctiveness ratings (DITC in Exp.1 and 3; DEV in Exp. 2 and 4) of male, female and all speakers in Exp. 1 - 4.

\begin{tabular}{|c|c|c|c|c|c|c|c|c|}
\hline \multirow{2}{*}{$\begin{array}{l}\text { Speaker } \\
\text { Sex }\end{array}$} & \multicolumn{4}{|c|}{ Vowels } & \multicolumn{4}{|c|}{ Sentences } \\
\hline & \multicolumn{2}{|c|}{ Attractiveness } & \multicolumn{2}{|c|}{ Distinctiveness } & \multicolumn{2}{|c|}{ Attractiveness } & \multicolumn{2}{|c|}{ Distinctiveness } \\
\hline & \multicolumn{4}{|c|}{ Experiment 1 (DITC) } & \multicolumn{4}{|c|}{ Experiment 3 (DITC) } \\
\hline Female & .191 & $(.296)$ & -.016 & $(.930)$ & -.078 & $(.673)$ & .328 & $(.067)$ \\
\hline Male & .267 & $(.140)$ & -.245 & $(.177)$ & .294 & $(.286)$ & -.076 & $(.677)$ \\
\hline \multirow[t]{2}{*}{ All } & .304 & $(.014)$ & -.211 & $(.095)$ & .142 & $(.263)$ & .137 & (.280) \\
\hline & \multicolumn{4}{|c|}{ Experiment 2 (DEV) } & \multicolumn{4}{|c|}{ Experiment 4 (DEV) } \\
\hline Female & .221 & $(.225)$ & .224 & $(.219)$ & -.052 & $(.775)$ & .026 & $(.886)$ \\
\hline Male & .084 & $(.648)$ & .049 & $(.791)$ & .119 & $(.516)$ & .153 & $(.405)$ \\
\hline All & .237 & $(.059)$ & .184 & $(.145)$ & .091 & $(.476)$ & .091 & (.476) \\
\hline
\end{tabular}



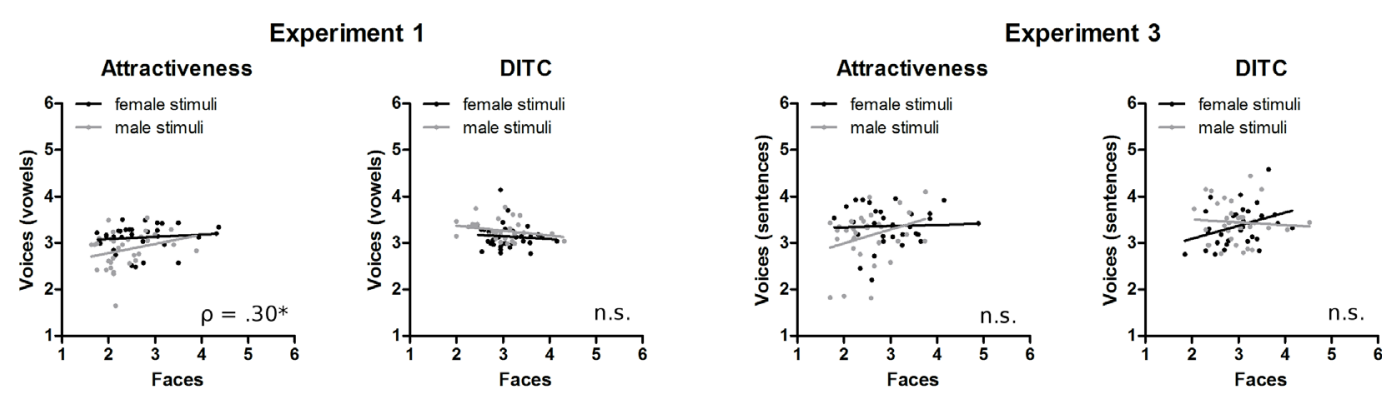

Experiment 2
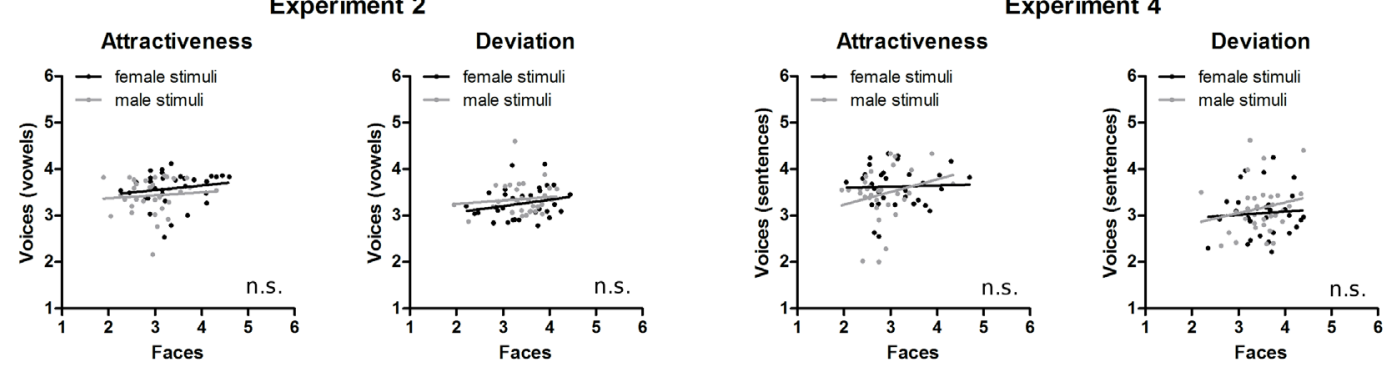

Fig 2. Between-domain correlations between mean ratings of faces and voices based on vowels (left: Exp. 1 and 2) and based on sentences (right: Exp. 3 and 4). Data are depicted separately for attractiveness and distinctiveness ratings (top: DITC-based distinctiveness; bottom: deviation-based distinctiveness) and for female (black) and male speakers (grey). Correlation coefficients $(\rho)$ are depicted overall, with asterisks indicating significance levels $\left({ }^{*} p<.05 ;{ }^{* *} p<.01 ;{ }^{* * *} p<.001\right)$. Note that facial and vocal attractiveness ratings for the same speakers were generally uncorrelated, with the exception of a small positive correlation when voice attractiveness ratings were based on vowel stimuli (Exp. 1).

When the above analyses were conducted separately for male and female speakers, we obtained virtually the same pattern of results (cf. Tables 1 and 2) with a few exceptions: in Exp. 1 the within-domain correlation between voice attractiveness and DITC was reduced to insignificance for male voices $(\rho[30]=-.32, p=.070)$, as were between-domain correlations for attractiveness when female and male stimuli were analyzed separately. In Exp. 3, the within-domain correlation between voice attractiveness and DITC was absent for male voices $(\rho[30]=.12, p=$ .515). However, it should be noted that these deviations from the overall pattern of results likely reflect a reduction of statistical power due to limited sample size. For the interested reader we report between-domain correlations also separately for male and female raters (supplemental Tables S12 and S13). In short, these analyses suggested similar results for both male and female raters. We also report statistical comparisons of the magnitudes of correlations for female vs. 
male speakers (supplemental Table S15). In short, these analyses did not suggest differences either.

Please note that we did not correct for multiple correlations. We considered that a problem of using an alpha adjustment for multiple tests (such as the Bonferroni correction) could have been that we likely would have missed small but important effects, such as small positive correlations between voice and face attractiveness in possible support of the honestsignal hypothesis. While our analysis strategy preserved maximal sensitivity of tests, it should be noted that our main finding of strong and negative correlations between attractiveness and DEV-based distinctiveness would easily survive Bonferroni correction for six tests, as performed for each experiment (as specified in Table 1).

\section{Discussion}

\subsection{Relationships between Attractiveness and Distinctiveness}

The present study is the first to demonstrate a systematic relationship between perceived attractiveness and distinctiveness in human voices. Here we found strong negative correlations between attractiveness and deviation-based distinctiveness (DEV) for voices when based both on vowels $(\rho=-.85)$ and on sentences $(\rho=-.87)$. This pattern was analogous to and, if anything, even stronger than the previously described negative correlation between attractiveness and deviation-based distinctiveness for faces (Exp. 2: $\rho=-.64$; Exp. 4: $\rho=-.74$ ). Overall, this pattern of findings provides strong support for an averageness account of attractiveness for both faces and voices (Bruckert et al., 2010; Langlois \& Roggman, 1990) when distinctiveness is assessed in a deviation-based manner. The present findings are important because they were obtained in the context of ratings for real stimuli, rather than for averaged "composite" stimuli. Note that averaging towards composites causes artifacts per se, such as smooth and symmetric visual patterns for faces, or increased harmonics-to-noise ratios for voices. We propose that, because such "nonaverage" features of digitally created composites have been shown to consistently contribute to perceived attractiveness (Bruckert et al., 2010; Rhodes, 2006), studies 
with natural individual stimuli that vary in perceived prototypicality or averageness are important to cross-validate findings obtained with composite stimuli.

Compared to these consistent findings of negative correlations between attractiveness and deviation-based distinctivness, the relationship with rated attractiveness was much less consistent for "in-the-crowd" based distinctiveness ratings. Specifically, while there was also a moderate negative correlation between attractiveness and "in-the-crowd" based distinctiveness (VITC) for voices when based on vowels (Exp. 1), this correlation was positive when based on sentences (Exp. 3). For faces, a marginally positive correlation between attractiveness and FITC was found in Exp. 1 (similar to Wiese et al., 2014), but this pattern was not replicated in Exp. 3 using the same stimuli and task. Taken together, our findings confirm that common deviationbased and "in-the-crowd"-based measures of distinctiveness (VITC and FITC) measure at least partially different constructs (Wiese et al., 2014), and extend those findings by showing that this is the case both for faces and for voices. For voices, the specific relationship between attractiveness and distinctiveness appears to depend on the type of utterance. Specifically, while simple vowel stimuli were rated as less attractive with increasing VITC distinctiveness (Exp. 1), in line with an averageness account, sentence stimuli (Exp. 3) were rated as more attractive with increasing VITC distinctiveness.

These differences between different types of utterances may generally be related to differences in duration and/or number of different phonemes (Andraszewicz et al., 2011; Ferdenzi et al., 2013). Whereas sentences carry much richer cues to attractiveness and distinctiveness, vowels are simple periodic utterances which are mainly influenced by "static" biophysical characteristics of individual speakers. VITC ratings for vowels could also differ from those for sentences due to a certain oddity of imagining someone saying a prolonged vowel sound in a crowd. Possibly related to this notion, an earlier study reported that perceived voice attractiveness and acoustic distance to mean (in terms of f0, F1) were correlated for a vowel $(/ \mathrm{a} /)$, but not for a word or sentence (Andraszewicz et al., 2011). Overall, ratings of attractiveness and VITC distinctiveness are likely based on partially different sets of acoustic 
cues, depending on their salience in a given utterance. The positive correlation between voice attractiveness and VITC distinctiveness is reminiscient of analogous findings for faces in previous research (Wiese et al., 2014) where it has been argued that DITC measures of distinctiveness may be distorted by cognitive heuristics. Accordingly, raters might be biased to think that they would surely spot a highly attractive person in the crowd, even when this might not be the case. Such an effect seems to generalize to voices in the present study, at least when ratings are based on sentence stimuli.

Unlike this previous study from face research (Wiese et al., 2014), however, the present data do not provide strong evidence for a positive correlation between attractiveness and FITC (Exp. 1 and 3). In our view, this could be related to the use of different face stimuli between studies: while the present stimuli originate from local volunteers and may not have contained a large number of extremely attractive faces (cf. Fig. 1), Wiese et al.'s (2014) face stimulus set was deliberatly designed to contain a large range of attractiveness levels, including exceptionally attractive faces. Possibly, these very attractive faces may have driven the previously reported correlation, as they would stand out of a crowd for being highly attractive.

In contrast to "in-the-crowd-based" measures of distinctiveness, correlations for deviation-based distinctiveness and attractiveness were highly consistent, and consistently negative across modalities and utterance types in the present study. This supports the notion that both faces and voices become increasingly attractive the more typical, i.e. the more average, they are perceived relative to prior personal experience (Bruckert et al., 2010; Langlois \& Roggman, 1990). At variance with this experience-based account of typicality, it has been argued recently that typicality ratings rather reflect stereotypes of what constitutes attractive and typical voices (Babel \& McGuire, 2015). In our view, this may be the case for tasks that do not further specify what typicality/distinctiveness is. However, it should be noted that our task explicitly invoked a memory component by asking participants to judge distinctiveness relative to the faces and voices they know. Given the different patterns of results for two types of 
distinctiveness measures, we believe that it is extremely important for future studies to specify exactly how typicality/distinctiveness was assessed.

Overall, while deviation-based measures gave rise to a highly consistent pattern of negative correlations with attractiveness across stimulus modalities and domains, inconsistent correlations were seen for attractiveness and DITC measures which may be distorted by subjective heuristics. This may indicate that deviation-based distinctiveness ratings are preferable over "in-the-crowd" based measures to assess distinctiveness in an unbiased manner.

\subsection{Relationships between Ratings for Faces and Voices of the Same Speakers}

The second aim of the present study was to provide a systematic assessment of relationships between ratings of attractiveness and distinctiveness for faces and voices from the same speakers. Positive correlations between independent ratings of faces and voices might be expected to the extent that (1) facial and vocal features are determined by the same underlying basis (e.g., genetic or hormonal), and (2) those features systematically influence perceptions under investigation (e.g., of attractiveness or distinctiveness). A common basis of vocal and facial attractiveness has been postulated by several studies (e.g. Collins \& Missing, 2003; Feinberg, 2008; Saxton et al., 2006). The present findings, however, consistently indicate that correlations between vocal and facial attractiveness are remarkably absent in the majority of the studied conditions, and small at best in one exception which we discuss below (Fig. 2). It needs to be noted that our negative findings for cross-domain relationships cannot have arisen because of insufficient variability in attractiveness in the present stimuli. This is because very strong and systematic negative correlations between attractiveness and deviation-based distinctiveness were actually observed within both the voice and the face domain. In that sense, the present

findings challenge the "honest signal account" of facial and vocal attractiveness, at least when investigated with complex naturalistic voice samples (Rhodes, 2006). It is worthwhile to consider the only exception to this pattern, in which we found a small but significant positive correlation between facial and vocal attractiveness, when simple vowels were used as voice 
samples. On the one hand, this finding potentially resolves discrepancies of our data to previous findings in which evidence for a correlation between facial and vocal attractiveness was found using similarly simple vocalizations (Collins \& Missing, 2003; Saxton et al., 2006). On the other hand, this result emphasizes the importance for researchers to consider the nature of the stimuli used to assess these relationships.

In that respect, prerequisites to find evidence for or against the honest signal hypothesis include that face and voice stimuli should be honest and undistorted representations of their owners genetic quality. We selected our face stimuli to be devoid of attractiveness-enhancing features such as make-up or jewellery. However, it may be more difficult to remove or standardize socio-cultural norms of attractiveness that are reflected in acquired speech patterns in the voice (Babel \& McGuire, 2015). In that sense, the present voice ratings to more naturalistic sentence stimuli may reflect in part cultural norms, rather than purely biophysically determined voice qualities. Accordingly, one explanation for the results found with simple vowels could be that these are relatively devoid of such socio-cultural cues, and thus may reflect genetic factors more "honestly" compared to more naturalistic and complex vocalizations. While this interesting possibility should be addressed in more detail in future research, we can conclude that correlations between vocal and facial attractiveness appear to be remarkably absent, at least when voices are presented in a naturalistic context akin to everyday communication.

As a possible limitation for both the present study and earlier research in this field (Abend et al., 2015; Collins \& Missing, 2003; Feinberg, 2008; Saxton et al., 2006), we assessed attractiveness and distinctiveness for static faces, and thus did not consider a possible role of dynamic facial information. To the extent that static and dynamic faces may be judged by different standards (Rubenstein, 2005), it remains possible that cross-domain correlations between facial and vocal attractiveness could be found for dynamic facial stimuli. In fact, one previous study emphasized the role of dynamic information for correlations between vocal and 
visual attractiveness, although this was not found consistently across different conditions (Lander, 2008). This issue warrants further investigations.

With respect to distinctiveness, facial and vocal ratings were uncorrelated for both types of distinctiveness ratings, suggesting no common basis for perceived distinctiveness. An interesting question for future research is how various measures of vocal distinctiveness could be related to one another. For instance, it would be instructive to assess in more detail how DITC and DEV are related with the actual recognisability of voices (for relevant research on faces, see Sommer, Heinz, Leuthold, Matt, \& Schweinberger, 1995; Vokey \& Read, 1992; Wiese et al., 2014), and to determine the acoustic stimulus parameters which underlie different aspects of perceived vocal distinctiveness (for relevant methods, see Skuk \& Schweinberger, 2014).

\section{Conclusions}

This study establishes, for the first time, substantial and systematic differences between deviation-based and "in-the-crowd" based measures of voice distinctiveness and their relationship to perceived attractiveness. Specifically, we found strong negative correlations between deviation-based distinctiveness and attractiveness, both for voices and faces, such that more distinctive speakers were perceived as less attractive. This supports the averageness account of attractiveness for both domains. By contrast, we found no such negative correlations between DITC and attractiveness, instead, correlations between DITC and attractiveness were marginally positive for faces, and significantly positive for voices when speakers uttered sentences, such that more distinctive voices were rated as more attractive. These findings strongly suggests that both measures of distinctiveness reflect at least partially different constructs, a finding which needs to be considered in future studies on vocal and facial distinctiveness.

An important finding of this research was that vocal and facial attractiveness ratings for the same speakers were remarkably uncorrelated, as were vocal and facial distinctiveness 
ratings. As a possible exception, a small positive correlation was observed for vocal and facial attractiveness ratings when vocal attractiveness ratings were based on simple vowel utterances which, while being rather unrepresentative of naturalistic speech, may be relatively less affected by sociocultural influences. Overall, these findings challenge the honest signal hypothesis of facial and vocal attractiveness at least in the context of ratings for relatively naturalistic speech, and emphasize the importance to consider in detail the nature of the stimuli used for this type research

\section{Acknowledgements}

This work was supported by the Deutsche Forschungsgemeinschaft (DFG; Grants ZA 745/1-1 and ZA 745/1-2; FOR1097). We cordially thank Constanze Mühl, Marie-Christin Perlich, Denise Müller and Mathias Riedel for help with stimulus editing and data acquisition. 


\section{References}

Abend, P., Pfluger, L. S., Koppensteiner, M., Coquerelle, M., \& Grammer, K. (2015). The sound of female shape: a redundant signal of vocal and facial attractiveness. Evolution and Human Behavior, 36(3), 174-181. doi:10.1016/j.evolhumbehav.2014.10.004

Andraszewicz, S., Yamagishi, J., King, S., \& Ieee. (2011). Vocal attractiveness of statistical speech synthesisers. 2011 IEEE International Conference on Acoustics, Speech, and Signal Processing, 5368-5371.

Babel, M., \& McGuire, G. (2015). Perceptual Fluency and Judgments of Vocal Aesthetics and Stereotypicality. Cognitive Science, 39(4), 766-787. doi:10.1111/cogs.12179

Babel, M., McGuire, G., \& King, J. (2014). Towards a More Nuanced View of Vocal Attractiveness. Plos One, 9(2). doi:10.1371/journal.pone.0088616

Boersma, P., \& Weenink, D. (2001). PRAAT, a system for doing phonetics by computer. Glot International, 5(9/10), 341-345.

Bruckert, L., Bestelmeyer, P., Latinus, M., Rouger, J., Charest, I., Rousselet, G. A., . . Belin, P. (2010). Vocal Attractiveness Increases by Averaging. Current Biology, 20(2), 116-120.

Collins, S. A. (2000). Men's voices and women's choices. Animal Behaviour, 60, 773-780.

Collins, S. A., \& Missing, C. (2003). Vocal and visual attractiveness are related in women. Animal Behaviour, 65, 997-1004.

Cunningham, M. R. (1986). Measuring the physical in physical attractiveness - quasiexperiments on the sociobiology of female facial beauty. Journal of Personality and Social Psychology, 50(5), 925-935. doi:10.1037/0022-3514.50.5.925

Feinberg, D. R. (2008). Are human faces and voices ornaments signaling common underlying cues to mate value? Evolutionary Anthropology, 17(2), 112-118.

Feinberg, D. R., DeBruine, L. M., Jones, B. C., \& Little, A. C. (2008). Correlated preferences for men's facial and vocal masculinity. Evolution and Human Behavior, 29(4), 233-241. doi:10.1016/j.evolhumbehav.2007.12.008 
Feinberg, D. R., DeBruine, L. M., Jones, B. C., \& Perrett, D. I. (2008). The role of femininity and averageness of voice pitch in aesthetic judgments of women's voices. Perception, $37(4), 615-623$.

Feinberg, D. R., Jones, B. C., DeBruine, L. M., Moore, F. R., Law Smith, M. J., Cornwell, R. E., , . Perrett, D. I. (2005). The voice and face of woman: One ornament that signals quality? Evolution and Human Behavior, 26(5), 398-408. doi:10.1016/j.evolhumbehav.2005.04.001

Fellowes, J. M., Remez, R. E., \& Rubin, P. E. (1997). Perceiving the sex and identity of a talker without natural vocal timbre. Perception \& Psychophysics, 59(6), 839-849.

Ferdenzi, C., Patel, S., Mehu-Blantar, I., Khidasheli, M., Sander, D., \& Delplanque, S. (2013). Voice attractiveness: Influence of stimulus duration and type. Behavior Research Methods, 45(2), 405-413. doi:10.3758/s13428-012-0275-0

Jones, B. C., Feinberg, D. R., DeBruine, L. M., Little, A. C., \& Vukovic, J. (2010). A domainspecific opposite-sex bias in human preferences for manipulated voice pitch. Animal Behaviour, 79(1), 57-62.

Kreiman, J., \& van Lancker Sidtis, D. (2011). Foundations of Voice Studies: An Interdisciplinary Approach to Voice Production and Perception (1st ed.). Chichester, UK: Wiley-Blackwell.

Lander, K. (2008). Relating visual and vocal attractiveness for moving and static faces. Animal Behaviour, 75, 817-822. doi:10.1016/j.anbehav.2007.07.001

Langlois, J. H., \& Roggman, L. A. (1990). Attractive faces are only average. Psychological Science, 1(2), 115-121. doi:10.1111/j.1467-9280.1990.tb00079.x

Lass, N. J., Hughes, K. R., Bowyer, M. D., Waters, L. T., \& Bourne, V. T. (1976). Speaker Sex Identification from Voiced, Whispered, and Filtered Isolated Vowels. Journal of the Acoustical Society of America, 59(3), 675-678.

Latinus, M., McAleer, P., Bestelmeyer, P. E., \& Belin, P. (2013). Norm-Based Coding of Voice Identity in Human Auditory Cortex. Current Biology, 23(12), 1075-1080. 
Linville, S. E. (1996). The sound of senescence. Journal of Voice, 10(2), 190-200.

Little, A. C., Connely, J., Feinberg, D. R., Jones, B. C., \& Roberts, S. C. (2011). Human preference for masculinity differs according to context in faces, bodies, voices, and smell. Behavioral Ecology, 22(4), 862-868. doi: 10.1093/beheco/arr061

Perrett, D. I., Lee, K. J., Penton-Voak, I., Rowland, D., Yoshikawa, S., Burt, D. M., . . . Akamatsu, S. (1998). Effects of sexual dimorphism on facial attractiveness. Nature, 394(6696), 884-887. doi:10.1038/29772

Rezlescu, C., Penton, T., Walsh, V., Tsujimura, H., Scott, S. K., \& Banissy, M. J. (2015). Dominant Voices and Attractive Faces: The Contribution of Visual and Auditory Information to Integrated Person Impressions. Journal of Nonverbal Behavior, 39(4), 355-370. doi:10.1007/s10919-015-0214-8

Rhodes, G. (2006). The evolutionary psychology of facial beauty Annual Review of Psychology (Vol. 57, pp. 199-226).

Rubenstein, A. J. (2005). Variation in perceived attractiveness - Differences between dynamic and static faces. Psychological Science, 16(10), 759-762. doi:10.1111/j.14679280.2005.01610.x

Saxton, T. K., Caryl, P. G., \& Roberts, S. C. (2006). Vocal and facial attractiveness judgments of children, adolescents and adults: The ontogeny of mate choice. Ethology, 112(12), 1179-1185. doi:10.1111/j.1439-0310.2006.01278.x

Schweinberger, S. R., Kawahara, H., Simpson, A. P., Skuk, V. G., \& Zäske, R. (2014). Speaker perception. Wiley Interdisciplinary Reviews-Cognitive Science, 5(1), 15-25.

Simpson, A. P. (2009). Phonetic differences between male and female speech. Language \& Linguistics Compass, 3, 621-640.

Skuk, V. G., \& Schweinberger, S. R. (2013). Gender differences in familiar voice identification. Hearing Research, 295, 131-140.

Skuk, V. G., \& Schweinberger, S. R. (2014). Influences of Fundamental Frequency, Formant Frequencies, Aperiodicity, and Spectrum Level on the Perception of Voice Gender. 
Journal of Speech Language and Hearing Research, 57(1), 285-296. doi:10.1044/1092$4388(2013 / 12-0314)$

Sommer, W., Heinz, A., Leuthold, H., Matt, J., \& Schweinberger, S. R. (1995). Metamemory, Distinctiveness, and Event-Related Potentials in Recognition Memory for Faces. Memory \& Cognition, 23(1), 1-11.

Valentine, T. (1991). A Unified Account of the Effects of Distinctiveness, Inversion, and Race in Face Recognition. Quarterly Journal of Experimental Psychology Section A-Human Experimental Psychology, 43(2), 161-204.

Valentova, J. V., Varella, M. A. C., Havlicek, J., \& Kleisner, K. (2017). Positive association between vocal and facial attractiveness in women but not in men: A cross-cultural study. Behavioural Processes, 135, 95-100.

Vokey, J. R., \& Read, J. D. (1992). Familiarity, memorability, and the effect of typicality on the recognition of faces. Memory \& Cognition, 20(3), 291-302. doi:10.3758/bf03199666

Wiese, H., Altmann, C. S., \& Schweinberger, S. R. (2014). Effects of attractiveness on face memory separated from distinctiveness: Evidence from event-related brain potentials. Neuropsychologia, 56, 26-36. doi:10.1016/j.neuropsychologia.2013.12.023

Willis, J., \& Todorov, A. (2006). First impressions: Making up your mind after a 100-ms exposure to a face. Psychological Science, 17(7), 592-598. doi:10.1111/j.14679280.2006.01750.x

Zuckerman, M., \& Miyake, K. (1993). The attractive voice - what makes it so. Journal of Nonverbal Behavior, 17(2), 119-135. doi:10.1007/bf01001960 
Table S1. Cronbach's alpha for voice and face attractiveness and distinctiveness ratings in Exp. 1 - 4.

\begin{tabular}{|c|c|c|c|c|}
\hline Rating dimension & Voices & Faces & Voices & Faces \\
\hline & \multicolumn{2}{|c|}{ Exp. 1 (vowels) } & \multicolumn{2}{|c|}{ Exp. 3 (sentences) } \\
\hline Attractiveness & .845 & .899 & .886 & .899 \\
\hline \multirow[t]{2}{*}{ Distinctiveness (DITC) } & .640 & .647 & .848 & .763 \\
\hline & \multicolumn{2}{|c|}{ Exp. 2 (vowels) } & \multicolumn{2}{|c|}{ Exp. 4 (sentences) } \\
\hline Attractiveness & .892 & .893 & .820 & .885 \\
\hline Distinctiveness (DEV) & .853 & .808 & .923 & .902 \\
\hline
\end{tabular}

Table S2. Spearman $\rho$ correlation coefficients (and p-values) for inter-vowel correlations between voice attractiveness and distinctiveness (VITC) ratings in Exp. 1.

\begin{tabular}{|c|c|c|c|c|c|c|c|c|c|c|}
\hline \multirow{3}{*}{$\begin{array}{c}\text { Exp. } 1 \\
\text { Attractiveness } \\
/ \mathrm{a} /\end{array}$} & \multicolumn{10}{|c|}{ Distinctiveness (VITC) } \\
\hline & \multicolumn{2}{|c|}{$/ \mathrm{a} /$} & \multicolumn{2}{|c|}{ /e/ } & \multicolumn{2}{|c|}{$/ \mathrm{i} /$} & \multicolumn{2}{|c|}{ /o/ } & \multicolumn{2}{|c|}{$/ \mathrm{u} /$} \\
\hline & -.19 & $(.128)$ & -.25 & $(.042)$ & -.29 & $(.020)$ & -.32 & $(.010)$ & -.31 & $(.012)$ \\
\hline /e/ & -.02 & $(.861)$ & -.40 & $(.001)$ & -.11 & $(.397)$ & -.22 & $(.086)$ & -.24 & $(.060)$ \\
\hline /i/ & -.17 & $(.190)$ & -.31 & $(.012)$ & -.30 & $(.014)$ & -.37 & $(.003)$ & -.22 & $(.084)$ \\
\hline /o/ & .06 & $(.661)$ & -.09 & $(.479)$ & -.15 & $(.246)$ & -.33 & $(.008)$ & -.35 & $(.005)$ \\
\hline$/ \mathrm{u} /$ & .01 & $(.967)$ & -.17 & $(.187)$ & .02 & $(.883)$ & -.28 & $(.027)$ & -.17 & $(.170)$ \\
\hline
\end{tabular}

Table S3. Spearman $\rho$ correlation coefficients (and p-values) for inter-vowel correlations between voice attractiveness and distinctiveness (DEV) ratings in Exp. 2.

Exp. 2

Attractiveness

\begin{tabular}{c} 
Attractiveness \\
\hline $\mathrm{a} /$ \\
$\mathrm{e} /$ \\
$\mathrm{i} /$ \\
$\mathrm{o} /$ \\
$\mathrm{h} /$
\end{tabular}

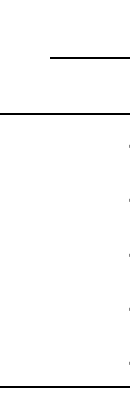

\begin{tabular}{|c|c|c|c|c|c|c|c|c|c|}
\hline \multicolumn{10}{|c|}{ Distinctiveness (DEV) } \\
\hline \multicolumn{2}{|c|}{$/ \mathrm{a} /$} & \multicolumn{2}{|c|}{ /e/ } & \multicolumn{2}{|c|}{ /i/ } & \multicolumn{2}{|c|}{ /o/ } & \multicolumn{2}{|c|}{$/ \mathrm{u} /$} \\
\hline-.74 & $(<.001)$ & -.35 & $(.005)$ & -.50 & $(<.001)$ & -.40 & $(.001)$ & -.33 & $(.008)$ \\
\hline-.27 & $(.034)$ & -.79 & $(<.001)$ & -.50 & $(<.001)$ & -.38 & $(.002)$ & -.34 & $(.007)$ \\
\hline-.51 & $(<.001)$ & -.52 & $(<.001)$ & -.79 & $(<.001)$ & -.42 & $(<.001)$ & -.35 & $(.005)$ \\
\hline-.33 & $(.007)$ & -.27 & $(.033)$ & -.42 & $(<.001)$ & -.60 & $(<.001)$ & -.45 & $(<.001)$ \\
\hline-.41 & $(<.001)$ & -.25 & $(.047)$ & -.41 & $(<.001)$ & -.49 & $(<.001)$ & -.57 & $(<.001)$ \\
\hline
\end{tabular}


Table S4. Spearman $\rho$ correlation coefficients (and p-values) for inter-sentence correlations between voice attractiveness and distinctiveness (VITC) ratings in Exp. 3.

\begin{tabular}{ccccccc} 
Exp. 3 & \multicolumn{5}{c}{ Distinctiveness (DITC) } \\
\cline { 2 - 7 } Attractiveness & \multicolumn{2}{c}{ sentence \#1 } & \multicolumn{3}{c}{ sentence \#2 } & \multicolumn{2}{c}{ sentence \#3 } \\
\hline sentence \#1 & .11 & $(.389)$ & .07 & $(.575)$ & -.07 & $(.568)$ \\
sentence \#2 & .28 & $(.027)$ & .31 & $(.014)$ & .11 & $(.399)$ \\
sentence \#3 & .41 & $(<.001)$ & .39 & $(.002)$ & .24 & $(.059)$ \\
\hline
\end{tabular}

Table S5. Spearman $\rho$ correlation coefficients (and p-values) for inter-sentence correlations between voice attractiveness and distinctiveness (DEV) ratings in Exp. 4.

\begin{tabular}{ccccccc} 
Exp. 4 & \multicolumn{5}{c}{ Distinctiveness (DEV) } \\
\cline { 2 - 7 } Attractiveness & \multicolumn{2}{c}{ sentence \#1 } & \multicolumn{3}{c}{ sentence \#2 } & \multicolumn{2}{c}{ sentence \#3 } \\
\hline sentence \#1 & -.88 & $(<.001)$ & -.60 & $(<.001)$ & -.51 & $(<.001)$ \\
sentence \#2 & -.68 & $(<.001)$ & -.85 & $(<.001)$ & -.64 & $(<.001)$ \\
sentence \#3 & -.45 & $(<.001)$ & -.57 & $(<.001)$ & -.75 & $(<.001)$ \\
\hline
\end{tabular}


Table S6. Spearman $\rho$ correlation coefficients (and p-values) for between-domain (vowels-face) correlations for attractiveness ratings in Exp. 1 and 2.

\begin{tabular}{ccccc} 
& \multicolumn{4}{c}{ Face Attractiveness } \\
\cline { 2 - 5 } $\begin{array}{c}\text { Voice } \\
\text { Attractiveness }\end{array}$ & \multicolumn{2}{c}{ Exp. 1 } & \multicolumn{2}{c}{ Exp. 2 } \\
\hline$/ \mathrm{a} /$ & .28 & $(.027)$ & .21 & $(.101)$ \\
$\mathrm{e} /$ & .14 & $(.284)$ & .21 & $(.098)$ \\
$\mathrm{i} /$ & .23 & $(.068)$ & .10 & $(.418)$ \\
$/ \mathrm{o} /$ & .20 & $(.122)$ & .06 & $(.617)$ \\
$/ \mathrm{u} /$ & .14 & $(.273)$ & .13 & $(.316)$ \\
\hline
\end{tabular}

Table S7. Spearman $\rho$ correlation coefficients (and p-values) for between-domain (vowels-face) correlations for distinctiveness ratings in Exp. 1 (DITC) and 2 (DEV).

\begin{tabular}{|c|c|c|c|c|}
\hline \multirow{3}{*}{$\begin{array}{c}\text { Voice } \\
\text { Distinctiveness } \\
/ \mathrm{a} /\end{array}$} & \multicolumn{4}{|c|}{ Face Distinctiveness } \\
\hline & \multicolumn{2}{|c|}{$\begin{array}{l}\text { Exp. } 1 \\
\text { (DITC) }\end{array}$} & \multicolumn{2}{|c|}{$\begin{array}{l}\text { Exp. } 2 \\
\text { (DEV) }\end{array}$} \\
\hline & -.09 & $(.491)$ & .01 & $(.927)$ \\
\hline /e/ & .02 & $(.880)$ & .17 & (.191) \\
\hline /i/ & -.06 & $(.660)$ & .11 & $(.367)$ \\
\hline$/ 0 /$ & -.26 & $(.037)$ & .18 & $(.154)$ \\
\hline$/ \mathrm{u} /$ & -.21 & $(.093)$ & .13 & $(.295)$ \\
\hline
\end{tabular}


Table S8. Spearman $\rho$ correlation coefficients (and p-values) for between-domain (sentences-face) correlations for attractiveness ratings in Exp. 3 and 4.

\begin{tabular}{ccccc} 
& \multicolumn{4}{c}{ Face Attractiveness } \\
\cline { 2 - 5 } Voice & \multicolumn{2}{c}{ Exp. 3 Exp. 4 } \\
Attractiveness & .20 & $(.117)$ & .20 & $(.108)$ \\
sentence \#1 & .10 & $(.422)$ & .07 & $(.579)$ \\
sentence \#2 & .18 & $(.164)$ & .05 & $(.717)$ \\
sentence \#3 &
\end{tabular}

Table S9. Spearman $\rho$ correlation coefficients (and p-values) for between-domain (sentences-face) correlations for distinctiveness ratings in Exp. 3 (DITC) and 4 (DEV).

\begin{tabular}{ccccc} 
& \multicolumn{3}{c}{ Face Distinctiveness } \\
\cline { 2 - 5 } Voice & \multicolumn{2}{c}{ Exp. 3 (DITC) } & \multicolumn{2}{c}{ Exp. 4 (DEV) } \\
\hline Distinctiveness & .13 & $(.294)$ & .11 & $(.376)$ \\
sentence \#1 & .05 & $(.707)$ & .09 & $(.475)$ \\
sentence \#2 & .18 & $(.157)$ & .05 & $(.723)$ \\
sentence \#3 & .18 &
\end{tabular}


Table S10. Female listeners: Spearman $\rho$ correlation coefficients (and p-values) for within-domain (voice and face) correlations between attractiveness and distinctiveness ratings (DITC in Exp.1 and 3; DEV in Exp. 2 and 4) depicted separately for female, male and all voices and faces in Exp. 1 - 4.

\begin{tabular}{|c|c|c|c|c|c|c|c|c|}
\hline \multirow[b]{2}{*}{$\begin{array}{l}\text { Speaker } \\
\text { Sex }\end{array}$} & \multicolumn{4}{|c|}{ Vowels } & \multicolumn{4}{|c|}{ Sentences } \\
\hline & \multicolumn{2}{|c|}{ Voices } & \multicolumn{2}{|c|}{ Faces } & \multicolumn{2}{|c|}{ Voices } & \multicolumn{2}{|c|}{ Faces } \\
\hline & \multicolumn{3}{|c|}{ Experiment 1 (DITC) } & & \multicolumn{4}{|c|}{ Experiment 3 (DITC) } \\
\hline Male & -.170 & $(.351)$ & .041 & $(.822)$ & .279 & $(.122)$ & .151 & $(.410)$ \\
\hline \multirow[t]{2}{*}{ All } & -.219 & $(.082)$ & .165 & $(.101)$ & .361 & $(.003)$ & .108 & (.396) \\
\hline & \multicolumn{3}{|c|}{ Experiment $2(\mathrm{DEV})$} & & \multicolumn{4}{|c|}{ Experiment 4 (DEV) } \\
\hline All & -.866 & $(<.001)$ & -.525 & $(<.001)$ & -.821 & $(<.001)$ & -.518 & $(<.001)$ \\
\hline
\end{tabular}

Table S11. Male listeners: Spearman $\rho$ correlation coefficients (and p-values) for within-domain (voice and face) correlations between attractiveness and distinctiveness ratings (DITC in Exp.1 and 3; DEV in Exp. 2 and 4) depicted separately for female, male and all voices and faces in Exp. 1 - 4.

\begin{tabular}{|c|c|c|c|c|c|c|c|c|}
\hline \multirow{2}{*}{$\begin{array}{l}\text { Speaker } \\
\text { Sex }\end{array}$} & \multicolumn{4}{|c|}{ Vowels } & \multicolumn{4}{|c|}{ Sentences } \\
\hline & \multicolumn{2}{|c|}{ Voices } & \multicolumn{2}{|c|}{ Faces } & \multicolumn{2}{|c|}{ Voices } & \multicolumn{2}{|c|}{ Faces } \\
\hline & \multicolumn{3}{|c|}{ Experiment 1 (DITC) } & & \multicolumn{4}{|c|}{ Experiment 3 (DITC) } \\
\hline Male & -.093 & $(.614)$ & .229 & $(.207)$ & .131 & $(.476)$ & -.195 & $(.284)$ \\
\hline All & -.406 & $(.001)$ & .205 & $(.104)$ & .193 & (.126) & -.136 & (.284) \\
\hline \multicolumn{5}{|c|}{ Experiment $2(\mathrm{DEV})$} & \multicolumn{4}{|c|}{ Experiment 4 (DEV) } \\
\hline All & -.681 & $(<.001)$ & -.627 & $(<.001)$ & -.836 & $(<.001)$ & -.743 & $(<.001)$ \\
\hline
\end{tabular}


Table S12. Female listeners: Spearman $\rho$ correlation coefficients (and p-values) for between-domain (voice-face) correlations depicted separately for attractiveness and distinctiveness ratings (DITC in Exp.1 and 3; DEV in Exp. 2 and 4) of male, female and all speakers in Exp. 1 - 4.

\begin{tabular}{|c|c|c|c|c|c|c|c|c|}
\hline \multirow[b]{2}{*}{$\begin{array}{l}\text { Speaker } \\
\text { Sex }\end{array}$} & \multicolumn{4}{|c|}{ Vowels } & \multicolumn{4}{|c|}{ Sentences } \\
\hline & \multicolumn{2}{|c|}{ Attractiveness } & \multicolumn{2}{|c|}{ Distinctiveness } & \multicolumn{2}{|c|}{ Attractiveness } & \multicolumn{2}{|c|}{ Distinctiveness } \\
\hline & \multicolumn{4}{|c|}{ Experiment 1 (DITC) } & \multicolumn{4}{|c|}{ Experiment 3 (DITC) } \\
\hline Male & .141 & $(.441)$ & -.298 & $(.097)$ & .191 & $(.294)$ & .024 & $(.895)$ \\
\hline \multirow[t]{2}{*}{ All } & .146 & $(.250)$ & -.242 & $(.054)$ & .294 & $(.018)$ & .089 & $(.482)$ \\
\hline & \multicolumn{4}{|c|}{ Experiment $2(\mathrm{DEV})$} & \multicolumn{4}{|c|}{ Experiment 4 (DEV) } \\
\hline All & .155 & $(.222)$ & .240 & $(.056)$ & .217 & $(.084)$ & .146 & $(.249)$ \\
\hline
\end{tabular}

Table S13. Male listeners: Spearman $\rho$ correlation coefficients (and p-values) for between-domain (voice-face) correlations depicted separately for attractiveness and distinctiveness ratings (DITC in Exp.1 and 3; DEV in Exp. 2 and 4) of male, female and all speakers in Exp. 1 - 4.

\begin{tabular}{|c|c|c|c|c|c|c|c|c|}
\hline \multirow{2}{*}{$\begin{array}{l}\text { Speaker } \\
\text { Sex }\end{array}$} & \multicolumn{4}{|c|}{ Vowels } & \multicolumn{4}{|c|}{ Sentences } \\
\hline & \multicolumn{2}{|c|}{ Attractiveness } & \multicolumn{2}{|c|}{ Distinctiveness } & \multicolumn{2}{|c|}{ Attractiveness } & \multicolumn{2}{|c|}{ Distinctiveness } \\
\hline & \multicolumn{4}{|c|}{ Experiment 1 (DITC) } & \multicolumn{4}{|c|}{ Experiment 3 (DITC) } \\
\hline Male & .456 & $(.009)$ & -.239 & $(.187)$ & .178 & $(.330)$ & -.262 & $(.148)$ \\
\hline \multirow[t]{2}{*}{ All } & .309 & (.013) & -.142 & $(.264)$ & .016 & (.899) & .063 & $(.623)$ \\
\hline & \multicolumn{4}{|c|}{ Experiment 2 (DEV) } & \multicolumn{4}{|c|}{ Experiment 4 (DEV) } \\
\hline All & .204 & $(.105)$ & .149 & $(.239)$ & .072 & (.572) & .077 & $(.545)$ \\
\hline
\end{tabular}


Table S14. Spearman $\rho$ correlation coefficients (and p-values) for within-domain (voice and face) correlations between attractiveness and distinctiveness ratings (DITC in Exp.1 and 3; DEV in Exp. 2 and 4) depicted separately for female, male and all voices and faces in Exp. 1 - 4, as well as comparisons between correlations for female vs. male stimuli according to Diedenhofen \& Musch (2015), with Fisher's Z (and p-values) in bold print.

\begin{tabular}{|c|c|c|c|c|c|c|c|c|}
\hline \multirow[b]{2}{*}{ Speaker Sex } & \multicolumn{4}{|c|}{ Vowels } & \multicolumn{4}{|c|}{ Sentences } \\
\hline & \multicolumn{2}{|c|}{ Voices } & \multicolumn{2}{|c|}{ Faces } & \multicolumn{2}{|c|}{ Voices } & \multicolumn{2}{|c|}{ Faces } \\
\hline & \multicolumn{4}{|c|}{ Experiment 1 (DITC) } & \multicolumn{4}{|c|}{ Experiment 3 (DITC) } \\
\hline Female & -.412 & $(.019)$ & .229 & $(.207)$ & .432 & $(.013)$ & -.071 & (.698) \\
\hline Male & -.324 & $(.070)$ & .128 & $(.484)$ & .119 & $(.515)$ & -.089 & $(.628)$ \\
\hline All & -.450 & $(<.001)$ & .207 & $(.101)$ & .275 & $(.028)$ & -.056 & $(.660)$ \\
\hline \multirow[t]{2}{*}{ Female vs. Male } & -0.388 & (.698) & 0.398 & (.691) & 1.305 & (.192) & -0.069 & (.945) \\
\hline & \multicolumn{4}{|c|}{ Experiment 2 (DEV) } & \multicolumn{4}{|c|}{ Experiment 4 (DEV) } \\
\hline Female & -.842 & $(<.001)$ & -.804 & $(<.001)$ & -.906 & $(<.001)$ & -.939 & $(<.001)$ \\
\hline Male & -.860 & $(<.001)$ & -.496 & $(.004)$ & -.804 & $(<.001)$ & -.580 & $(.001)$ \\
\hline All & -.853 & $(<.001)$ & -.639 & $(<.001)$ & -.866 & $(<.001)$ & -.744 & $(<.001)$ \\
\hline Female vs. Male & 0.248 & $(.804)$ & -2.155 & $(.031)$ & -1.504 & $(.133)$ & -4.063 & $(<.001)$ \\
\hline
\end{tabular}


Table S15. Spearman $\rho$ correlation coefficients (and p-values) for between-domain (voice-face) correlations depicted separately for attractiveness and distinctiveness ratings (DITC in Exp.1 and 3; DEV in Exp. 2 and 4) of male, female and all speakers in Exp. 1 - 4, as well as comparisons between correlations for female vs. male stimuli according to Diedenhofen \& Musch (2015), with Fisher's Z (and p-values) in bold print.

\begin{tabular}{|c|c|c|c|c|c|c|c|c|}
\hline \multirow[b]{2}{*}{ Speaker Sex } & \multicolumn{4}{|c|}{ Vowels } & \multicolumn{4}{|c|}{ Sentences } \\
\hline & \multicolumn{2}{|c|}{ Attractiveness } & \multicolumn{2}{|c|}{ Distinctiveness } & \multicolumn{2}{|c|}{ Attractiveness } & \multicolumn{2}{|c|}{ Distinctiveness } \\
\hline & \multicolumn{4}{|c|}{ Experiment 1 (DITC) } & \multicolumn{4}{|c|}{ Experiment $3(\mathrm{DITC})$} \\
\hline Female & .191 & $(.296)$ & -.016 & $(.930)$ & -.078 & $(.673)$ & .328 & $(.067)$ \\
\hline Male & .267 & $(.140)$ & -.245 & $(.177)$ & .294 & $(.286)$ & -.076 & $(.677)$ \\
\hline All & .304 & $(.014)$ & -.211 & $(.095)$ & .142 & $(.263)$ & .137 & $(.280)$ \\
\hline \multirow[t]{2}{*}{ Female vs. Male } & -0.306 & $(.760)$ & 0.891 & $(.372)$ & -1.451 & $(.147)$ & 1.587 & (.113) \\
\hline & \multicolumn{4}{|c|}{ Experiment 2 (DEV) } & \multicolumn{4}{|c|}{ Experiment 4 (DEV) } \\
\hline Female & .221 & $(.225)$ & .224 & $(.219)$ & -.052 & $(.775)$ & .026 & $(.886)$ \\
\hline Male & .084 & $(.648)$ & .049 & $(.791)$ & .119 & $(.516)$ & .153 & $(.405)$ \\
\hline All & .237 & $(.059)$ & .184 & $(.145)$ & .091 & $(.476)$ & .091 & $(.476)$ \\
\hline Female vs. Male & 0.535 & $(.593)$ & 0.681 & $(.496)$ & -0.654 & $(.513)$ & -0.488 & $(.625)$ \\
\hline
\end{tabular}

References

Diedenhofen, B., \& Musch, J. (2015). cocor: A Comprehensive Solution for the Statistical Comparison of Correlations. Plos One, 10(4). doi:10.1371/journal.pone.0121945 